\title{
Intravenous Dextroamphetamine and Brain Glucose Metabolism
}

\author{
Monique Ernst, M.D., Ph.D., Alan J. Zametkin, M.D., John Matochik, Ph.D., \\ Mark Schmidt, M.D., Peter H. Jons, B.A., Laura L. Liebenauer, M.A., \\ Kristina K. Hardy, B.A., and Robert M. Cohen, M.D., Ph.D.
}

This study reports the effects of intravenous dextroamphetamine on cerebral glucose metabolism assayed by positron emission tomography (PET) and [fluorine18]fluorodeoxyglucose (FDG) in 13 healthy adults during the performance of a continuous visual attention task. Two FDG PET scans were performed within a single experimental session. The first scan was preceded by the injection of placebo and the second scan by the injection of $0.15 \mathrm{mg} / \mathrm{kg}$ dextroamphetamine. Global and normalized regional glucose metabolic rates ( $r C M R g l c)$ were examined as a function of pharmacological challenge and subjective experience. Subcortical, limbic, frontal, and cerebellar
rCMRglc significantly increased after dextroamphetamine, whereas $r C M R g l c$ of the temporal cortex significantly decreased. Physiological and self-report measures of subjective states showed the expected alterations. These rCMRglc changes reflect both the direct pharmacological effect of dextroamphetamine on monoaminergic neurotransmitter systems as well as enhancement of the activation of the neural network mediating the performance of the continuous attention task.

[Neuropsychopharmacology 17:391-401, 1997]

Published by Elsevier Science Inc.
KEY WORDS: Stimulant; PET; Dextroamphetamine; Attention; FDG; Imaging

Dextroamphetamine administration produces clear increases of brain glucose metabolism in animals using autoradiography with $\mathrm{C}^{14}$-2-deoxyglucose (Berntman et al. 1978; Porrino et al. 1984; Wechsler et al. 1979). Studies in humans are inconsistent and indicate either small decreases or no effects of stimulants on glucose metabolism or cerebral blood flow (Matochik et al. 1993, 1994; Ernst et al. 1994; Wolkin et al. 1987; Daniel et al. 1991; Kahn et al. 1989; Mathew and Wilson 1985; Wang et al.

From the Laboratory of Cerebral Metabolism, National Institute of Mental Health, National Institutes of Health, Bethesda, Maryland.

Address correspondence to: Monique Ernst, M.D., Ph.D., Brain Imaging Center, NIDA-IRP, 5500 Nathan Shock Drive, Baltimore, MD 21224.

Received September 3, 1996; revised December 16, 1996; accepted February 13, 1997.
1994; Metz et al. 1991). Few studies report increases in cerebral blood flow associated with the administration of stimulants (Devous et al. 1995; Lou et al. 1984, 1989). The discrepancies in these findings may reflect species differences, imaging methods (positron emission tomography (PET), single photon emission computerized tomography (SPECT), or autoradiography), indices of brain activity (cerebral blood flow, glucose utilization), doses and routes of administration of stimulant, duration of data collection ( 1 to $4 \mathrm{~min}$ in blood flow studies, 30 to $90 \mathrm{~min}$ in glucose metabolism studies), latency between the administration of the stimulant and the collection of brain activity data (no time lag to $3 \mathrm{~h}$ ), or status of subjects (psychiatric condition; at rest or performing a task). The last factor has been exploited in an attempt to clarify neural circuits involved in cognitive function (Friston et al. 1992; Kapur et al. 1994; Dolan et al. 1995; Mattay et al. 1996) and in psychopathology (Daniel et al. 1991). 
Three PET studies with [fluorine-18]fluorodeoxyglucose (FDG) assessed dextroamphetamine effect on cerebral glucose metabolic rates (Wolkin et al. 1987; Matochik et al. 1993, 1994). These studies used oral administration of clinically relevant doses of stimulant in psychiatric patients. Because of the relatively few changes in regional glucose metabolic rates in these studies, the intravenous route of administration would appear to be more sensitive in the detection of changes in brain function, since autoradiographic studies of deoxyglucose in animals, using parenteral routes, have been successful in identifying robust effects of dextroamphetamine (Berntman et al. 1978; Porrino et al. 1984; Wechsler et al. 1979). A preliminary study of PET and FDG from our laboratory reported few changes in regional glucose metabolic rates after intravenous administration of dextroamphetamine at $0.15 \mathrm{mg} / \mathrm{kg}$ in eight adults with attention-deficit hyperactivity disorder (ADHD) (Ernst et al. 1994). Even though the behavioral effects of stimulants are similar in normal and ADHD individuals (Rapoport et al. 1980), sensitivity of neural networks to stimulants may be different. Three other studies of intravenous dextroamphetamine or methylphenidate assessed cerebral blood flow with SPECT (Mathew and Wilson 1985) or PET (Kahn et al. 1989; Wang et al. 1994). The interpretation of these studies is confounded because of the possible vasoactive effects of stimulants, in contrast to measures of glucose metabolism, which have been shown to be virtually independent of cerebral blood flow (Sokoloff 1984).

The present study tests the hypothesis that the intravenous administration of dextroamphetamine at 0.15 $\mathrm{mg} / \mathrm{kg}$ in normal healthy adults would replicate the findings of increases of cerebral glucose metabolism, particularly in dopamine-rich regions and in those regions mediating the performance of the attention task.

\section{SUBJECTS AND METHODS}

\section{Subjects}

The sample consisted of 13 normal healthy volunteers (male $/$ female $=5 / 8 ; 28.3 \pm 8.3$ years) who were medication-free for a minimum of 3 weeks before the study. Screening included a complete physical and laboratory medical work-up. Subjects were psychiatrically evaluated by means of a semi-structured diagnostic interview (Schedule for Affective Disorders and Schizophrenia, life-long version; Endicott and Spitzer 1978). Exclusion criteria were the presence of any axis I or II DSM-III-R psychiatric disorders (American Psychiatric Association 1987) or of any medical problems including neurological deficits, history of head trauma with loss of consciousness, and cardiac or blood pressure abnormalities.

\section{Design}

The study consisted of a 3-h FDG PET session. Each session included a first scan after a 3 min intravenous infusion of placebo (normal saline), and a second scan after a $3 \mathrm{~min}$ intravenous infusion of $0.15 \mathrm{mg} / \mathrm{kg}$ dextroamphetamine. Order of drug (placebo first, dextroamphetamine second) was fixed because of the potential carryover effects of dextroamphetamine. The design of the study is shown in Figure 1. Subjects were instructed that they would receive placebo or dextroamphetamine before one or the other FDG PET scans. Except for the physician responsible for the study, the research team was blind as to the nature of the injection.

A continuous visual performance task (CPT) was used during both 30-min uptake periods of the FDG tracer, primarily as a way to control for internal and external stimulation. Subjects were trained on the CPT $1 \mathrm{~h}$ before the PET study. The attention task consisted of the random presentation of degraded numbers. Subjects were asked to press a button each time they saw a " 1 " followed by a "9." An auditory distractor was introduced to increase the difficulty of the task. While performing the task, subjects listened through headphones to a recording of numbers read in random order. CPT scores included number of correctly identified responses within a specified latency (hits), and number of missed responses (misses). The tracer FDG was infused intravenously over a 1 -min period at a dose of $3 \mathrm{mCi}$ for the first scan and $5 \mathrm{mCi}$ for the second scan.

Heart rate and blood pressure were continuously monitored throughout the study via a pressure transducer on the arterial line. The means of two measures at 10 and 5 min pre-dextroamphetamine (baseline) and at $5 \mathrm{~min}$ and $10 \mathrm{~min}$ post-dextroamphetamine, respectively, were used for analysis. Two standardized ratings were used to assess affective states: (1) 28-item How I Feel questionnaire (HIF) (Rapoport et al. 1980), and the (2) Modified Spielberger State Anxiety Scale (STAI) (Spielberger et al. 1970).

\section{Positron Emission Tomography (PET)}

A 7-slice brain scanner (Scanditronix PC-1024-7B) with an in-plane resolution of $5.2 \mathrm{~mm}$ full width at half maximum (FWHM), interslice interval of $13.8 \mathrm{~mm}$, and an axial resolution of $10 \mathrm{~mm}$ FWHM was used. Each scan included three sets of 7 slices, which were later interleaved to create a set of 21 planes at $4.6-\mathrm{mm}$ intervals. Three 10-min emission acquisitions, each of seven image planes, were obtained at $0 \mathrm{~mm}, 4.5 \mathrm{~mm}$ and $9 \mathrm{~mm}$ above the cantho-meatal line. Attenuation correction was calculated from transmission scans at each position, using a rotating germanium $\left({ }^{68} \mathrm{Ge}\right)$ pin, obtained before the first emission scan and before and after the second emission scan (Carson et al. 1988). Tracer input 


\section{TIMELINE OF PAIRED FDG SCANS}

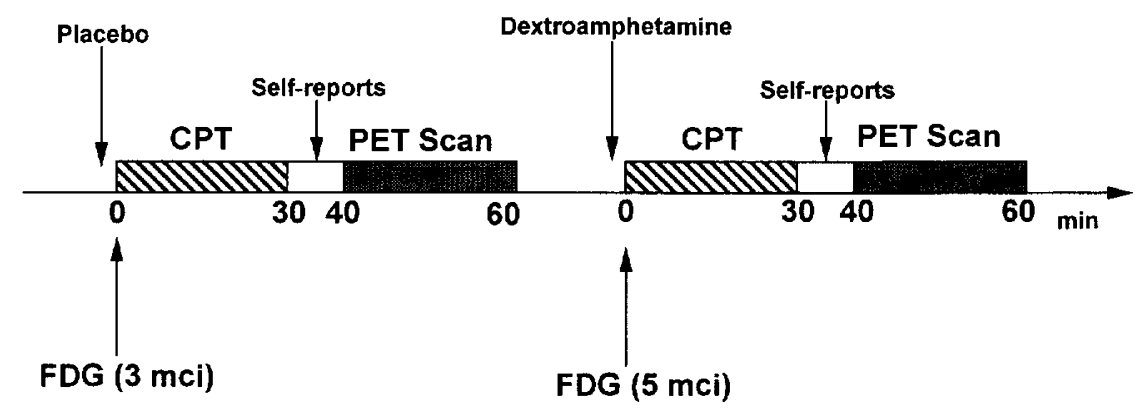

Figure 1. Timeline of the PET session.

curves were calculated from arterial blood samples drawn through a catheter inserted in the radial artery.

Raw pixel values were converted to glucose metabolic rates in mg of glucose per $100 \mathrm{~g}$ of tissue per min, using a modification of the Sokoloff operational equation (Brooks 1982; Phelps et al. 1979) and a lumped constant of 0.418 (Huang et al. 1980). The second emission scan was corrected for residual activity from the first scan, using the plasma activity curve and plasma activity immediately before the second FDG injection (Brooks et al. 1987). Five transaxial planes were selected from each set of 21 planes to match a standard set containing neuroanatomic regions of interest identified by using the atlas of Matsui and Hirano (1978). Standardized templates of 60 regions of interest (ROIs) were applied to the five selected planes and adjusted manually for individual anatomical differences. Image analysis of regional rates of glucose metabolism were calculated by an independent rater blind to condition (placebo, dextroamphetamine). Global metabolic rates (average absolute CMRglc of all examined cortical gray-matter regions), and normalized regional CMRglc (rCMRglc = absolute regional CMRglc divided by global CMRglc) were examined. Normalized values were used to minimize the effects on regional metabolism of interindividual variability in global metabolism.

\section{Analysis of the Results}

To limit the number of statistical tests, the 60 ROIs were combined into seven large regions: frontal, parietal, temporal, and occipital cortices, limbic region (rCMRglc mean of cingulate and hippocampus), subcortical area (rCMRglc mean of thalamus, caudate, and putamen), and cerebellar region.

A multivariate analysis of variance with repeated measures (MANOVA) assessed the effects of drug (placebo versus dextroamphetamine) and region on rCMRglc of the seven large regions. Significance was based on the interaction term [drug * region]. If the interaction term was significant, then paired $t$-tests were used for simple tests. The significance level was set at 0.05 and trend at 0.10 .

When rCMRglc of a large region was significantly altered by dextroamphetamine, the individual contributions of the ROIs composing the large region were assessed by means of a MANOVA. Here again, significances were judged on the interaction terms [drug * region]. Paired $t$-tests were performed on the individual ROIs of any large region whose MANOVA was statistically significant.

Changes in behavior were assessed by means of paired $t$-tests. Correlations of significant changes between clinical variables and rCMRglc were calculated by means of Pearson correlation coefficients.

All changes represented the subtractions of measures during scan 2 from measures during scan 1 (scan 2-scan 1).

\section{RESULTS}

\section{Sample (Table 1)}

Characteristics of the subjects are presented in Table 1. Performance on the visual attention task was high at baseline (91\% correct responses), resulting in a ceiling

Table 1. Sample Characteristics $(n=13)$

\begin{tabular}{lc}
\hline & Baseline \\
\cline { 2 - 2 } & Mean \pm SD \\
\hline Age (years) & $28.3 \pm 8.3$ \\
Sex (male/female) & $5 / 8$ \\
SES & $55.2 \pm 29.8$ \\
IQ full scale & $106.0 \pm 12.6$ \\
Spielberger (STAI) & $17.8 \pm 8.0$ \\
CPT-hits & $89.3 \pm 10.1$ \\
CPT-misses & $6.0 \pm 8.7$ \\
\hline
\end{tabular}


Table 2. Glucose Metabolic Rates in Combined Regions $(n=13)$

\begin{tabular}{lccccc}
\hline & & & \multicolumn{2}{c}{$\begin{array}{c}\text { Comparison } \\
(\boldsymbol{n}=\mathbf{1 3})\end{array}$} \\
\cline { 5 - 6 } & $\begin{array}{c}\text { Scan-1 } \\
\text { Mean (SD) }\end{array}$ & $\begin{array}{c}\text { Scan-2 } \\
\text { Mean (SD) }\end{array}$ & $\boldsymbol{t}$ & $\boldsymbol{p}$ \\
\hline Global CMRglc & & & & \\
$\quad$ (mg/100g/min) & $12.70(2.02)$ & $13.06(3.02)$ & 0.58 & NS \\
Subcortical & $0.98(0.08)$ & $1.04(0.07)$ & 3.34 & 0.006 \\
Frontal & $1.03(0.02)$ & $1.03(0.02)$ & 2.44 & 0.031 \\
Temporal & $0.96(0.03)$ & $0.94(0.03)$ & 2.66 & 0.021 \\
Limbic & $0.85(0.05)$ & $0.89(0.05)$ & 3.42 & 0.005 \\
Cerebellum & $0.71(0.06)$ & $0.79(0.08)$ & 6.63 & 0.0001 \\
Parietal & $0.95(0.03)$ & $0.95(0.03)$ & 0.19 & NS \\
Occipital & $0.98(0.07)$ & $1.00(0.09)$ & 1.51 & NS \\
\hline
\end{tabular}

effect and making it difficult to detect an effect of dextroamphetamine challenge on attention in the task.

\section{Glucose Metabolic Rates (Table 2; Figure 2)}

Global CMRglc. Global CMRglc was not significantly altered by dextroamphetamine (CMRglc change $=0.36$ $\mathrm{mg} / 100 \mathrm{~g} / \mathrm{min}, d f=12, t=0.58, \mathrm{NS})$.
Normalized rCMRglc. The overall MANOVA of the seven large regions showed significant interaction of region * drug $(d f=6,66$, Wilks' lambda $=0.09, \mathrm{~F}=8.93$, $p=.0001)$.

Paired $t$-tests showed that the rCMRglc of four of the seven large regions were significantly increased by dextroamphetamine: Subcortical ( $d f=12, t=3.34, p=$ .0059), limbic $(d f=12, t=3.42, p=.0051)$, cerebellar $(d f=$ $12, t=6.63, p=.0001)$ and frontal regions $(d f=12, t=$ $2.44, p=.031)$; rCMRglc of one region, the temporal cortex, was significantly reduced $(d f=12, t=-2.66$, $p=.021$ ). Figure 2 shows individual changes of rCMRglc in the five regions significantly altered by dextroamphetamine challenge.

MANOVAs of two of the five large regions altered by dextroamphetamine showed significant interactions between ROIs and drug (subcortical: $d f=2,24$, Wilks' lambda $=0.55, \mathrm{~F}=4.70, p=.02$; and temporal: $d f=$ 3,36 , Wilks' lambda $=0.46, F=3.53, p=.02$ ). Subsequent paired $t$-tests showed that among the ROIs composing the subcortical region, the caudate nucleus was the only one whose increase in rCMRglc reached statistical significance ( $d f=12, t=3.76, p=.003)$; among the ROIs composing the temporal region, the superior ante-

\section{SUBCORTICAL rCMRgIC}

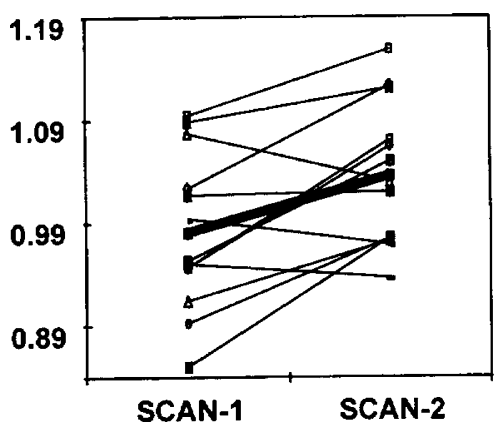

FRONTAL rCMRgIC

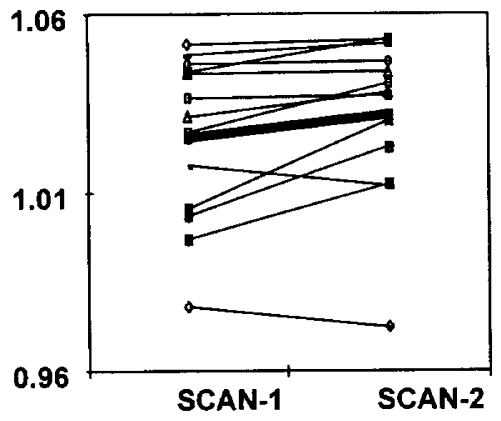

TEMPORAL rCMRglc

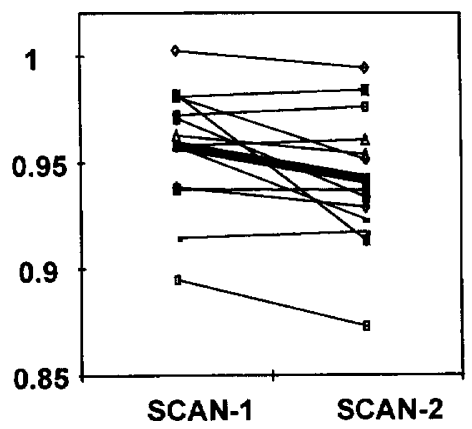

LIMBIC rCMRgIC

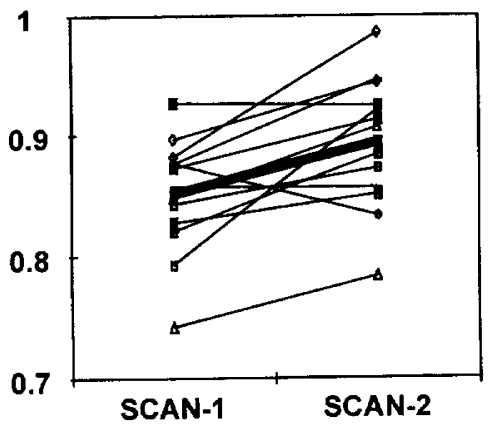

\section{CEREBELLAR rCMRglc}

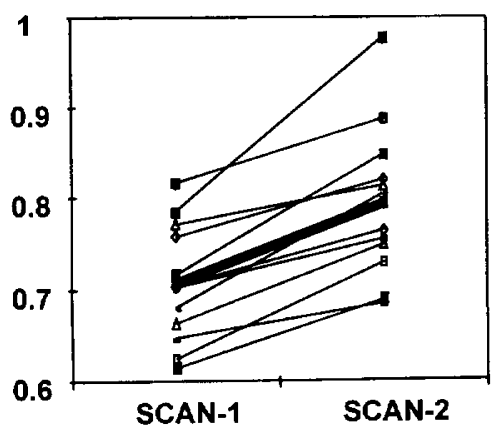

Figure 2. Individual values $(n=13)$ of normalized rCMRglc of the five regions significantly altered by dextroamphetamine during scan1 (placebo) and during scan 2 (dextroamphetamine). $y$-axis: normalized rCMRglc is the ratio of regional to global glucose metabolic rate. The thick line connects the mean point of rCMRglc during scan 1 (placebo) to the mean point of rCMRglc during scan 2 (dextroamphetamine). 
rior temporal ROI was the only one whose decrease in rCMRglc reached statistical significance ( $d f=12, t=$ $3.04, p=.01$ ). Table 3 lists the rCMRglc changes by ROIs.

\section{Physiological and Behavioral Scores (Table 4)}

Dextroamphetamine significantly increased blood pressure (diastolic blood pressure: $d f=12, t=5.29, p=.0002$; systolic blood pressure: $d f=12, t=6.24, p=.0001$ ) and did not affect heart rate. Subjects showed reduction in tiredness (HIF-tired: $d f=12, \mathrm{t}=4.50, p=.0007$ ), and increases in good mood (HIF-good mood: $d f=12, t=2.74$, $p=.018$ ), in feeling unlike themselves (HIF-weird: $d f=$ $12, t=-4.06, p=.0016$ ), more talkative (HIF-talk: $d f=$ $12, t=3.74, p=.003$ ), and thinking faster (HIF-thoughts: $d f=12, t=4.42, p=.0008$ ). Self-reports of anxiety, atten-

Table 3. Normalized rCMRglc in All ROIs Examined

\begin{tabular}{|c|c|c|c|c|c|c|}
\hline \multirow{2}{*}{$\begin{array}{l}\text { Large Regions } \\
\text { ROI }\end{array}$} & \multicolumn{3}{|c|}{$\begin{array}{c}\text { Scan 1 } \\
(\text { Mean } \pm \text { SD) }\end{array}$} & \multicolumn{3}{|c|}{$\begin{array}{c}\text { Scan } 2 \\
(\text { Mean } \pm \text { SD })\end{array}$} \\
\hline & Left Side & Medial & Right Side & Left Side & Medial & Right Side \\
\hline \multicolumn{7}{|l|}{ Frontal } \\
\hline A plane anterior medial frontal & \multicolumn{3}{|c|}{$1.01 \pm 0.08$} & \multicolumn{3}{|c|}{$0.99 \pm 0.08$} \\
\hline A plane lateral anterior frontal & $1.05 \pm 0.05$ & & $1.04 \pm 0.08$ & $1.04 \pm 0.06$ & & $1.01 \pm 0.06$ \\
\hline A plane lateral posterior frontal & $1.04 \pm 0.07$ & & $1.04 \pm 0.05$ & $1.01 \pm 0.06$ & & $1.02 \pm 0.08$ \\
\hline B plane anterior medial frontal & \multicolumn{3}{|c|}{$0.97 \pm 0.05$} & \multicolumn{3}{|c|}{$0.99 \pm 0.06$} \\
\hline B plane lateral anterior frontal & $1.02 \pm 0.06$ & & $1.01 \pm 0.07$ & $1.03 \pm 0.04$ & & $1.04 \pm 0.06$ \\
\hline B plane lateral posterior frontal & \multirow{2}{*}{\multicolumn{3}{|c|}{$0.97 \pm 0.05$}} & $1.07 \pm 0.06$ & & $1.06 \pm 0.06$ \\
\hline C plane anterior medial frontal & & & & \multicolumn{3}{|c|}{$0.98 \pm 0.07$} \\
\hline C plane lateral anterior frontal & $1.05 \pm 0.05$ & & $1.06 \pm 0.07$ & $1.06 \pm 0.06$ & & $1.07 \pm 0.08$ \\
\hline C plane lateral posterior frontal & $1.06 \pm 0.08$ & & $1.14 \pm 0.08$ & $1.05 \pm 0.07$ & & $1.10 \pm 0.08$ \\
\hline D plane anterior medial frontal & \multicolumn{3}{|c|}{$0.99 \pm 0.04^{\mathrm{c}}$} & \multicolumn{3}{|c|}{$1.03 \pm 0.05$} \\
\hline D plane lateral anterior frontal & $1.04 \pm 0.06$ & & $1.07 \pm 0.05$ & $1.05 \pm 0.07$ & & $1.09 \pm 0.05$ \\
\hline D plane lateral posterior frontal & $1.01 \pm 0.05$ & & $1.04 \pm 0.07$ & $1.01 \pm 0.10$ & & $1.03 \pm 0.08$ \\
\hline E plane anterior medial frontal & \multicolumn{3}{|c|}{$0.97 \pm 0.05$} & \multicolumn{3}{|c|}{$0.98 \pm 0.05$} \\
\hline E plane lateral anterior frontal & $1.01 \pm 0.05$ & & $1.05 \pm 0.07$ & $1.02 \pm 0.08$ & & $1.04 \pm 0.07$ \\
\hline E plane lateral posterior frontal & $0.93 \pm 0.07$ & & $0.96 \pm 0.05$ & $0.96 \pm 0.05$ & & $0.98 \pm 0.08$ \\
\hline \multicolumn{7}{|l|}{ Parietal } \\
\hline A plane superior medial parietal & \multicolumn{3}{|c|}{$1.04 \pm 0.07$} & \multicolumn{3}{|c|}{$1.01 \pm 0.06$} \\
\hline A plane lateral parietal & $1.00 \pm 0.08$ & & $1.01 \pm 0.08$ & $1.01 \pm 0.05$ & & $1.03 \pm 0.06$ \\
\hline B plane lateral parietal & $0.96 \pm 0.10$ & & $0.94 \pm 0.05$ & $0.98 \pm 0.08$ & & $0.99 \pm 0.07$ \\
\hline B plane lateral rolandic & $0.89 \pm 0.10$ & & $0.91 \pm 0.06$ & $0.89 \pm 0.12$ & & $0.90 \pm 0.07$ \\
\hline C plane lateral parietal & $0.95 \pm 0.06^{a}$ & & $0.96 \pm 0.06$ & $0.99 \pm 0.07$ & & $0.98 \pm 0.08$ \\
\hline C plane lateral sylvian & $0.99 \pm 0.05^{c}$ & & $1.00 \pm 0.07$ & $0.91 \pm 0.07$ & & $0.98 \pm 0.08$ \\
\hline \multicolumn{7}{|l|}{ Temporal } \\
\hline D plane lateral anterior temporal & $0.99 \pm 0.09$ & & $1.02 \pm 0.06^{a}$ & $0.94 \pm 0.06$ & & $0.97 \pm 0.07$ \\
\hline D plane lateral medial temporal & $1.00 \pm 0.05$ & & $0.99 \pm 0.06$ & $1.00 \pm 0.06$ & & $0.99 \pm 0.07$ \\
\hline D plane lateral posterior temporal & $0.94 \pm 0.05$ & & $0.87 \pm 0.05$ & $0.93 \pm 0.04$ & & $0.89 \pm 0.08$ \\
\hline E plane lateral temporal & $0.92 \pm 0.08$ & & $0.94 \pm 0.07$ & $0.87 \pm 0.08$ & & $0.95 \pm 0.07$ \\
\hline \multicolumn{7}{|l|}{ Subcortical } \\
\hline D plane lateral thalamus & $1.00 \pm 0.09^{b}$ & & $1.04 \pm 0.11$ & $1.10 \pm 0.09$ & & $1.02 \pm 0.13$ \\
\hline D plane lateral caudate & $0.98 \pm 0.09^{b}$ & & $0.97 \pm 0.14^{b}$ & $1.09 \pm 0.11$ & & $1.10 \pm 0.16$ \\
\hline D plane lateral anterior putamen & $0.94 \pm 0.13$ & & $0.90 \pm 0.18^{a}$ & $0.96 \pm 0.13$ & & $0.98 \pm 0.22$ \\
\hline D plane lateral posterior putamen & $0.89 \pm 0.10$ & & $0.88 \pm 0.13$ & $0.86 \pm 0.08$ & & $0.91 \pm 0.15$ \\
\hline \multicolumn{7}{|l|}{ Occipital } \\
\hline A plane medial occipital & \multicolumn{3}{|c|}{$1.08 \pm 0.12$} & \\
\hline B plane medial occipital & \multirow{2}{*}{\multicolumn{3}{|c|}{$\begin{array}{l}1.05 \pm 0.11 \\
1.15 \pm 0.13\end{array}$}} & & $1.06 \pm 0.11$ & \\
\hline C plane medial occipital & & & & & $1.16 \pm 0.15$ & \\
\hline C plane lateral occipital & $0.86 \pm 0.07$ & & $0.84 \pm 0.07$ & $0.87 \pm 0.08$ & & $0.90 \pm 0.13$ \\
\hline Limbic & & & & & & \\
\hline B plane medial cingulate & & $1.00 \pm 0.07$ & & & $1.05 \pm 0.11$ & \\
\hline E plane hippocampus & $0.77 \pm 0.05^{a}$ & & $0.78 \pm 0.07^{a}$ & $0.82 \pm 0.06$ & & $0.82 \pm 0.07$ \\
\hline Cerebellar & & & & & & \\
\hline F plane lateral cerebellar & $0.71 \pm 0.07^{c}$ & & $0.70 \pm 0.07^{c}$ & $0.80 \pm 0.09$ & & $0.79 \pm 0.09$ \\
\hline
\end{tabular}

Statistical significances (paired $t$-tests; scan 1 versus scan 2):

" $p<.05$

$b_{p}<.01$;

$c p<.001$ 
Table 4. Physiological and Self-Report Measures Significantly Altered by Dextroamphetamine Challenge

\begin{tabular}{lcccc}
\hline & Scan-1 & Scan-2 & \multicolumn{2}{c}{$\begin{array}{c}\text { Comparison } \\
(\boldsymbol{n}=\mathbf{1 3})\end{array}$} \\
\cline { 4 - 5 } & Mean (SD) & Mean (SD) & $t$ & \multicolumn{1}{c}{$p$} \\
\hline Systolic-BP (first 10 min) & $128.7(10.6)$ & $164.9(20.1)$ & 6.24 & 0.0001 \\
Diastolic-BP (first 10 min) & $69.9(9.6)$ & $81.8(12.8)$ & 5.29 & 0.0002 \\
HIF-tired & $1.23(0.93)$ & $0.15(0.38)$ & 4.50 & 0.0007 \\
HIF-good mood & $1.00(0.82)$ & $1.77(1.01)$ & 2.74 & 0.018 \\
HIF-more talkative & $0.08(0.28)$ & $1.15(0.99)$ & 3.74 & 0.003 \\
HIF-thinking fast & $0.23(0.66)$ & $1.23(0.83)$ & 4.42 & 0.0008 \\
HIF-weird & $0.46(0.66)$ & $1.69(1.03)$ & 4.06 & 0.002 \\
\hline
\end{tabular}

tion, and restlessness as well as CPT performance were not significantly altered by dextroamphetamine.

\section{Correlations of Significant Changes (Scan 2-Scan 1) between Behavioral Measures and rCMRglc (Table 5)}

None of the rCMRglc changes correlated significantly with changes in blood pressure. Among the correlations between the seven large regions and the five behavioral variables significantly altered by dextroamphetamine (HIF measures of tiredness, feeling good, feeling unlike themselves, talkative, thinking fast), only one was significant; reduction in tiredness was associated with increased rCMRglc in the frontal cortex (HIF-tired: $d f=$ $12, r=-0.55, p=.05$ ).

\section{DISCUSSION}

\section{Behavioral Effects}

As expected, dextroamphetamine produced strong blood pressure increases, mood elevation, heightened arousal (less tired, more talkative, thinking faster) and no changes in self-report levels of restlessness and attention. CPT performance was not influenced by dextroamphetamine. Interpretation of the lack of changes in CPT performance warrants some caution because of the presence of a ceiling effect in this group of healthy normal volunteers. CPT performance was nearly optimal during scan 1 and could not improve significantly in scan 2. None of the subjects were fidgety or felt restless during scan 1; therefore, they could not report reduction in these variables during dextroamphetamine.

\section{CMRglc Effects}

Although the intravenous administration of dextroamphetamine failed to alter global CMRglc, it did produce significant regional changes in selective brain regions:
rCMRglc increased in subcortical, limbic, cerebellar, and frontal regions and decreased in temporal region. The effects of dextroamphetamine, a nonspecific monoaminergic agonist, reflect both its direct pharmacological impact on monoaminergic neurotransmitters systems as well as a selective response of the functional neural network activated by the performance on an attention task. Therefore, the relative distribution of the various neurotransmitters and the functional role of individual brain regions are important to consider in the interpretation of the results. Unfortunately, the design of this study does not permit to clearly disentangle the pharmacological effects of the drug from its influence on the task-related neural activation.

Two possible sources of artifacts need to be addressed: (1) alterations of rCMRglc in the regions involved in movement (subcortical, frontal, and cerebellar areas) are not likely to be due to changes in motor activity after dextroamphetamine administration. Number and rate of button pressings and accuracy of performance during the attention task were similar between pre- and post-dextroamphetamine scans, and subjects did not report changes in restlessness or fidgetiness between scans. (2) It is unlikely for the rCMRglc changes to be solely an effect of order (placebo first and dextroamphetamine second). A recent study of two independent samples of 10 healthy volunteers and eight adults with ADHD using similar experimental PET conditions as those in the present study showed that global CMRglc did not change between paired scans during placebo condition (Schmidt et al. 1996). Normalized rCMRglc differed significantly in two of 60 ROIs in the group of normal volunteers (the middle medial region in plane $\mathrm{A}$ increased, and the right parietal region in plane A decreased from scan 1 to scan 2) and in three of 60 ROIs in the group of ADHD subjects (the middle cingulate region increased, and the left anterior frontal region in plane $\mathrm{A}$ and right posterior frontal region in plane $C$ decreased from scan 1 to scan 2) (Schmidt et al. 1996). The significantly altered rCMRglc did not overlap between groups and did not exceed chance findings. 
Table 5. Pearson Correlation Coefficients of Changes in Normalized Regional Glucose Metabolic Rates of ROIs (scan 2 scan 1) with Statistically Significant Changes (scan 2 - scan 1) in Clinical Variables

\begin{tabular}{|c|c|c|c|c|c|c|c|}
\hline $\begin{array}{l}\text { Large Regions } \\
\text { ROI }\end{array}$ & $\begin{array}{c}\text { Diastolic } \\
\text { BP }\end{array}$ & $\begin{array}{c}\text { Systolic } \\
\text { BP }\end{array}$ & $\begin{array}{c}\text { HIF } \\
\text { Good mood }\end{array}$ & $\begin{array}{c}\text { HIF } \\
\text { Talk a lot }\end{array}$ & $\begin{array}{c}\text { HIF } \\
\text { Think fast }\end{array}$ & $\begin{array}{c}\text { HIF } \\
\text { Feel weird }\end{array}$ & $\begin{array}{c}\text { HIF } \\
\text { Feel tired }\end{array}$ \\
\hline Global CMRglc & -0.02 & -0.10 & -0.47 & 0.12 & 0.34 & $0.57^{a}$ & -0.39 \\
\hline \multicolumn{8}{|l|}{ Frontal } \\
\hline A plane anterior medial frontal & 0.04 & -0.02 & 0.12 & -0.27 & -0.50 & $-0.56^{a}$ & 0.26 \\
\hline A plane left anterior frontal & 0.22 & -0.17 & -0.23 & -0.30 & -0.50 & -0.51 & -0.04 \\
\hline A plane right anterior frontal & 0.51 & 0.18 & 0.02 & -0.10 & 0.03 & 0.18 & -0.22 \\
\hline A plane left posterior frontal & 0.35 & 0.02 & -0.17 & 0.52 & 0.09 & 0.46 & $0.64^{a}$ \\
\hline A plane right posterior frontal & 0.05 & 0.12 & 0.06 & 0.34 & 0.11 & 0.18 & $-0.62^{a}$ \\
\hline B plane anterior medial frontal & -0.02 & -0.17 & -0.15 & -0.16 & $-0.56^{a}$ & -0.33 & 0.06 \\
\hline B plane left anterior frontal & -0.24 & -0.00 & -0.23 & 0.18 & -0.09 & -0.13 & 0.29 \\
\hline B plane right anterior frontal & 0.01 & 0.09 & -0.11 & -0.46 & -0.20 & 0.43 & 0.10 \\
\hline B plane left posterior frontal & 0.34 & 0.07 & -0.01 & 0.12 & -0.53 & -0.16 & 0.18 \\
\hline B plane right posterior frontal & -0.13 & -0.19 & -0.38 & -0.31 & -0.40 & 0.09 & -0.04 \\
\hline C plane anterior medial frontal & 0.34 & $0.64^{a}$ & 0.11 & 0.41 & $0.55^{a}$ & 0.18 & -0.08 \\
\hline C plane left anterior frontal & 0.25 & 0.33 & 0.22 & 0.17 & 0.51 & -0.23 & -0.46 \\
\hline C plane right anterior frontal & 0.29 & 0.30 & 0.21 & -0.50 & 0.07 & 0.30 & -0.12 \\
\hline C plane left posterior frontal & -0.41 & -0.33 & 0.24 & -0.12 & -0.01 & 0.12 & 0.36 \\
\hline $\mathrm{C}$ plane right posterior frontal & -0.39 & -0.30 & 0.05 & -0.18 & 0.53 & $0.64^{a}$ & -0.47 \\
\hline D plane anterior medial frontal & -0.10 & 0.30 & -0.04 & $0.66^{a}$ & 0.39 & -0.05 & -0.00 \\
\hline D plane left anterior frontal & $0.55^{a}$ & $0.56^{a}$ & 0.28 & 0.30 & -0.07 & 0.09 & -0.34 \\
\hline $\mathrm{D}$ plane right anterior frontal & -0.41 & -0.14 & -0.27 & -0.04 & 0.08 & 0.08 & -0.15 \\
\hline D plane left posterior frontal & 0.06 & $0.57^{a}$ & -0.01 & $0.55^{a}$ & 0.15 & -0.07 & 0.23 \\
\hline D plane right posterior frontal & -0.33 & $-0.85^{c}$ & -0.06 & -0.02 & -0.07 & -0.06 & 0.22 \\
\hline E plane anterior medial frontal & -0.21 & 0.22 & -0.06 & 0.21 & 0.46 & -0.36 & -0.07 \\
\hline E plane left anterior frontal & 0.18 & 0.08 & 0.37 & -0.18 & 0.18 & -0.07 & -0.28 \\
\hline E plane right anterior frontal & 0.25 & 0.00 & 0.07 & 0.06 & -0.11 & 0.03 & $-0.55^{a}$ \\
\hline E plane left posterior frontal & -0.53 & -0.07 & 0.08 & 0.16 & 0.36 & -0.16 & 0.03 \\
\hline E plane right posterior frontal & 0.25 & -0.28 & -0.01 & -0.47 & -0.42 & 0.27 & $-0.59^{a}$ \\
\hline \multicolumn{8}{|l|}{ Parietal } \\
\hline A plane superior medial parietal & 0.16 & 0.35 & -0.11 & -0.11 & -0.08 & 0.02 & -0.35 \\
\hline A plane left parietal & 0.06 & 0.13 & 0.08 & 0.14 & 0.47 & 0.16 & -0.04 \\
\hline A plane right parietal & -0.03 & -0.42 & 0.06 & -0.22 & 0.33 & 0.16 & -0.26 \\
\hline B plane left parietal & $0.59^{a}$ & 0.24 & 0.04 & 0.11 & -0.17 & 0.01 & -0.07 \\
\hline $\mathrm{B}$ plane right parietal & 0.14 & -0.11 & -0.18 & 0.25 & 0.08 & 0.54 & 0.09 \\
\hline B plane left rolandic & -0.30 & -0.02 & 0.31 & -0.13 & -0.15 & -0.33 & $0.56^{a}$ \\
\hline B plane right rolandic & -0.22 & 0.06 & 0.19 & 0.08 & 0.09 & 0.02 & 0.43 \\
\hline$C$ plane left parietal & 0.04 & -0.03 & -0.23 & $0.69^{b}$ & 0.44 & -0.00 & -0.35 \\
\hline$C$ plane right parietal & -0.36 & -0.33 & 0.17 & 0.00 & $0.62^{a}$ & 0.34 & 0.17 \\
\hline C plane left sylvian & -0.00 & 0.05 & 0.22 & -0.07 & -0.45 & -0.19 & -0.26 \\
\hline$C$ plane right sylvian & 0.03 & -0.21 & 0.30 & 0.12 & -0.01 & $-0.60^{a}$ & 0.10 \\
\hline \multicolumn{8}{|l|}{ Temporal } \\
\hline D plane left anterior temporal & -0.07 & 0.10 & 0.26 & 0.27 & 0.04 & -0.03 & -0.18 \\
\hline D plane right anterior temporal & 0.17 & $0.59^{a}$ & -0.32 & -0.09 & 0.14 & 0.35 & -0.31 \\
\hline D plane left medial temporal & -0.40 & -0.28 & -0.17 & 0.10 & 0.11 & 0.12 & 0.51 \\
\hline D plane right medial temporal & -0.14 & -0.48 & -0.42 & -0.09 & 0.09 & 0.32 & 0.17 \\
\hline D plane left posterior temporal & -0.27 & -0.09 & -0.34 & $0.82^{c}$ & $0.57^{a}$ & 0.06 & 0.32 \\
\hline D plane right posterior temporal & -0.22 & 0.07 & 0.02 & $-0.74^{b}$ & -0.37 & -0.11 & -0.28 \\
\hline E plane left temporal & 0.29 & 0.08 & -0.21 & 0.32 & 0.36 & -0.01 & 0.32 \\
\hline E plane right temporal & 0.28 & -0.05 & 0.18 & -0.36 & -0.24 & 0.33 & 0.03 \\
\hline \multicolumn{8}{|l|}{ Subcortical } \\
\hline D plane left thalamus & 0.46 & $0.77^{b}$ & 0.01 & 0.05 & 0.22 & -0.01 & -0.51 \\
\hline D plane right thalamus & -0.01 & 0.21 & 0.13 & 0.12 & -0.20 & 0.14 & -0.05 \\
\hline D plane left cuadate & 0.04 & -0.04 & 0.00 & 0.11 & 0.17 & 0.08 & -0.15 \\
\hline D plane right caudate & 0.17 & 0.02 & -0.14 & 0.31 & 0.20 & -0.31 & -0.21 \\
\hline D plane left anterior putamen & 0.07 & 0.27 & 0.06 & 0.21 & 0.17 & -0.05 & 0.17 \\
\hline D plane right anterior putamen & 0.16 & -0.10 & -0.43 & -0.06 & -0.48 & -0.35 & -0.15 \\
\hline D plane left posterior putamen & -0.27 & -0.37 & -0.29 & -0.27 & $-0.59^{a}$ & 0.13 & -0.11 \\
\hline D plane right posterior putamen & -0.09 & -0.42 & -0.01 & -0.50 & -0.35 & -0.30 & -0.21 \\
\hline
\end{tabular}


Table 5. (continued)

\begin{tabular}{|c|c|c|c|c|c|c|c|}
\hline $\begin{array}{l}\text { Large Regions } \\
\text { ROI }\end{array}$ & $\begin{array}{c}\text { Diastolic } \\
\text { BP }\end{array}$ & $\begin{array}{c}\text { Systolic } \\
\text { BP }\end{array}$ & $\begin{array}{c}\text { HIF } \\
\text { Good mood }\end{array}$ & $\begin{array}{c}\text { HIF } \\
\text { Talk a lot }\end{array}$ & $\begin{array}{c}\text { HIF } \\
\text { Think fast }\end{array}$ & $\begin{array}{c}\text { HIF } \\
\text { Feel weird }\end{array}$ & $\begin{array}{c}\text { HIF } \\
\text { Feel tired }\end{array}$ \\
\hline \multicolumn{8}{|l|}{ Occipital } \\
\hline A plane medial occipital & 0.38 & -0.27 & 0.15 & -0.00 & -0.30 & -0.10 & 0.00 \\
\hline B plane medial occipital & -0.14 & -0.08 & -0.23 & 0.10 & -0.19 & -0.22 & 0.42 \\
\hline C plane medial occipital & 0.00 & -0.13 & 0.14 & -0.19 & -0.09 & -0.27 & -0.30 \\
\hline \multicolumn{8}{|l|}{ Limbic } \\
\hline B plane medial cingulate & 0.09 & -0.28 & -0.15 & 0.37 & 0.11 & -0.20 & 0.32 \\
\hline E plane left hippocampus & -0.53 & -0.14 & -0.24 & -0.08 & -0.08 & -0.16 & 0.12 \\
\hline E plane right hippocampus & -0.26 & 0.16 & -0.08 & 0.48 & 0.28 & 0.20 & 0.30 \\
\hline \multicolumn{8}{|l|}{ Cerebellar } \\
\hline F plane left cerebellar & -0.33 & -0.00 & -0.03 & -0.34 & 0.03 & 0.08 & 0.33 \\
\hline F plane right cerebellar & -0.50 & 0.24 & 0.02 & -0.30 & -0.20 & -0.16 & 0.14 \\
\hline $\begin{array}{l}\text { Significance values as follows: } \\
{ }^{a} p<.05 \\
{ }^{b} p<.01 \\
c_{p}<.001 \\
\text { HIF = "How I feel" questionnai }\end{array}$ & & & & & & & \\
\hline
\end{tabular}

\section{Subcortical and Frontal rCMRglc}

Frontal and subcortical regions are examined jointly because of their strong functional connections (Gerfen 1992; Alexander and Crutcher 1990).

Dextroamphetamine induces the release of dopamine from presynaptic storage vesicles and blocks reuptake of dopamine (Kuczenski and Segal 1989). The subsequent enhanced dopaminergic activity in the striatum modulates cortical inputs via thalamic projections. Two proposed functional models of this modulatory role describe the coexistence of opposite actions of dopaminergic release in the striatum. (1) Parallel pathways of striatothalamic projections include direct inhibitory and indirect disinhibitory afferents (Gerfen 1992; Alexander and Crutcher 1990). (2) At the cellular level, extracellular dopamine concentration exerts a negative tonic feedback onto dopaminergic nerve terminals, and intrasynaptic dopamine concentration phasically activates post-synaptic nerve fibers (Grace 1995). Therefore, the direction of cortical regulation by the dopaminergic system can be either excitatory or inhibitory and depends on the state of the subject, such as level of arousal and sensorimotor activation. The elevation of rCMRglc in the frontal cortex measured in the present study may reflect an excitatory striatal influence that results in facilitating performance of the attention task. The frontal cortex, particularly the lateral prefrontal region, has been shown to be selectively activated during the performance of a sustained attention task (Cohen et al. 1988, 1992).

Frontal activation was not as robust as subcortical activation ( $p=.03$ vs. $p=.006$ ). This difference may reflect the competing influences in the frontal cortex of inhibitory and excitatory effects of dopamine or the contribution of other factors, such as a noradrenergic cortical inhibition produced by dextroamphetaminerelated norepinephrine release (West et al. 1995). That frontal rCMRglc increase was associated, at a trend level, with a reduction in "feeling tired" suggests that the integrated effect of dextroamphetamine on this region translates into alterations of subjective experience more readily than do changes in other brain regions that did not correlate with any changes in self-reports.

\section{Limbic rCMRglc}

Limbic regions are involved in emotional arousal and cognition, mainly memory (hippocampus) and attention (cingulate) and receive important noradrenergic innervation. Neural activity in the hippocampus has also been shown to be modulated by dopaminergic (Glowinski et al. 1984; Mantz et al. 1988; Mogenson and Yim, 1991) and serotoninergic input (Coop and McNaugghton 1991; Sprouse and Aghajanian 1988). Any of the monoaminergic neurotransmitter systems as well as any combinations of their effects may mediate the activation of the limbic system observed in this study, since amphetamine enhances the release of dopamine, norepinephrine, and serotonin from storage vesicles in nerve terminals (Weiner 1980; Kuczenski and Segal 1989). The activation of these regions may mediate the strong subjective behavioral changes (good mood, more talkative, less tired) reported by the subjects, which are typical of the stimulant-related feeling of "high." Motivation was proposed as the component of attention modulated by limbic/paralimbic areas (Tucker et al. 1995; Derryberry and Tucker 1990). Although motivation was not directly measured in this study, sub- 
jects reported a change of attitude toward the performance of the task, from being bored and irritated before dextroamphetamine to feeling challenged and positive after dextroamphetamine.

\section{Cerebellar rCMRglc}

Activation of the cerebellar cortex was the most robust effect of dextroamphetamine. This effect was surprising because of the weak monoaminergic innervation in this region, particularly dopaminergic innervation. Therefore, rCMRglc cerebellar changes are likely to be indirect and may be partly context-dependent, that is, related to the changes in the cognitive demands of the performance task. A growing body of evidence supports a role of the cerebellum in cognitive function (Decety et al. 1990; Kim et al. 1994; Daum and Ackermann 1995; Leiner et al. 1993). The cerebellum was shown to contribute to the neural network that mediates voluntary shift of attention between sensory modalities (Akshoomoff and Courchesne, 1992). This finding concurs with the subjects' reports of becoming able to pay attention to external and internal stimuli without experiencing difficulty in performing the attention task during the dextroamphetamine condition. PET studies have also demonstrated that the cerebellum contributed to practice-related learning and automatization of performance (Daum and Ackermann 1995; Massion and Rispal-Padel 1986). Even though performance level did not change between scan 1 and scan 2 , due to a ceiling effect, subjects reported more ease in carrying out the attention task. The neural mechanism by which dextroamphetamine activates the cerebellum, as well as the cognitive-behavioral translation of this effect, need to be clarified.

\section{Temporal rCMRglc}

The functional significance of the decreased rCMRglc in the temporal region after dextroamphetamine is likely to be linked to the role of the temporal cortex in auditory processing (Rumsey et al. 1992). Although not formally recorded, subjects reported that they were able to ignore the auditory distractor of the attention task (random numbers delivered through earphones) much more easily after dextroamphetamine. Monoamines have been shown to inhibit spontaneous background neural firing while amplifying selectively the neural responses to a specific sensory stimulus (Foote et al. 1975; Johnson et al. 1983). In this case, the auditory stimuli were treated more successfully as background noise after stimulant through a more effective inhibition of the temporal neural activity subserving auditory processes. Dextroamphetamine, by releasing monoamines, may have facilitated the processing of relevant stimuli at the expense of irrelevant ones. This effect has been observed previ- ously in studies in humans, where a selective enhancement of task-dependent activation of regional cerebral blood flow was reported after administration of dextroamphetamine using SPECT and PET methodologies (Daniel et al. 1991; Mattay et al. 1996).

\section{Relationships between Behavioral and rCMRglc Changes}

The results showed no significant relationships between behavioral changes and rCMRglc alterations in the large regions. The ceiling effect in the cognitive performance on the CPT prevented the exploration of an association between change in performance and change in rCMRglc with dextroamphetamine. The absence of correlation between physiological (blood pressure) and rCMRglc changes indicates a dissociation of peripheral from central effects of dextroamphetamine on the nervous system. Associations between smaller, more functionally defined brain regions may have occurred. However, because of the large number of correlation tests ( 60 ROIs $\times 6$ behavioral measures), any correction for family-wise error would have made it impossible to detect statistical significances, and any significant result without correction for multiple tests would not have been interpretable.

In conclusion, dextroamphetamine infused over 3 $\mathrm{min}$ at $0.15 \mathrm{mg} / \mathrm{kg}$, produced the expected behavioral changes, and was associated with selective regional alterations in brain glucose metabolism. These changes reflect both the direct pharmacological action of the drug (release of monoamines) and the interaction of the pharmacological effect with the performance of an attention task. Neural networks involved in attention processing were activated (subcortical structures, frontal cortex, cerebellum), as were those involved in motivational and emotional states (limbic/paralimbic system). Finally, activity in the region involved in auditory and language processing was inhibited. This pattern of changes is consistent with a role of dextroamphetamine in elevating mood as well as in improving signal-tonoise ratio in processing information. Functional brain imaging studies of dextroamphetamine challenge may help examine the functional capacity of neural networks to modulate attention in various psychiatric disorders with known impairments in attentional skills, such as attention-deficit hyperactivity disorder, schizophrenia, or autism.

\section{ACKNOWLEDGMENTS}

We thank the PET technologists and Chemistry Department of Nuclear Medicine, NIH Clinical Center for assistance in performing the study. 


\section{REFERENCES}

Alexander GE, Crutcher MD (1990): Functional architecture of basal ganglia circuits: Neural substrates of parallel processing. TINS 13(7):266-271

American Psychiatric Association (1987): Diagnostic and Statistical Manual of Mental Disorders, 3rd ed. rev. (DSM-IIIR). Washington, DC, American Psychiatric Association

Akshoomoff NA, Courchesne E (1992): A new role for the cerebellum in cognitive operations. Behav Neurosci 106: $731-738$

Berntman L, Carlsson S, Hagerdal M, Siesjo BK (1978): Circulatory and metabolic effects in the brain induced by amphetamine sulphate. Acta Physiol Scand 102:310-323

Brooks RA (1982): Alternative formula for glucose utilization using labeled deoxyglucose. J Nucl Med 2:538-539

Brooks RA, DiChiro G, Zukerberg BW, Bairamian D, Larson SM (1987): Test-retest studies of cerebral glucose metabolism using fluorine-18 deoxyglucose: Validation of method. J Nucl Med 28:53-59

Carson RE, Daube-Witherspoon ME, Green MV (1988): A method for postinjection PET transmission measurements with a rotating source. J Nucl Med 29:1558-1567

Cohen RM, Semple WE, Gross M, Holcomg HH, Dowling MS, Nordahl TE (1988): Functional localization of sustained attention: Comparison to sensory stimulation in the absence of instruction. Neuropsychiatry Neuropsychol Behav Neurol 1(1):3-20

Cohen RM, Semple WE, Gross M, King AC, Nordahl TE (1992): Metabolic brain pattern of sustained auditory discrimination. Exp Brain Res 92:165-172

Coop CF, McNaugghton N (1991): Buspirone affects hippocampal rhythmical slow activity through serotonin $1 \mathrm{~A}$ rather than $D_{2}$ receptors. Neuroscience 40:169-174

Daniel DG, Weinberger DR, Jones DW, Zigun JR, Coppola R, Handel S, Bigelow LB, Goldberg TE, Berman KF, Kleinman JE (1991): The effect of amphetamine on regional cerebral blood flow during cognitive activation in schizophrenia. J Neurosci 11:1907-1917

Daum I, Ackermann H (1995): Cerebellar contributions to cognition. Behav Brain Res 67:201-210

Decety J, Sjoholm H, Ryding E, Stenberg G, Ingvar D (1990): The cerebellum participates in mental activity: Tomographic measurements of regional cerebral blood flow. Brain Res 535:313-317

Derryberry D, Tucker DM (1990): The adaptive base of the neural hierarchy: Elementary motivational controls on network function. Nebr Symp Motiv 38:289-342

Devous MD Sr, Trivedi MH, Gong W, Harris TS, Rush AJ (1995): RCBF changes in limbic and limbically innervated brain regions in normal controls following oral amphetamine challenge. J Cereb Blood Flow 15:S829

Dolan RJ, Fletcher P, Frith CD, Friston KI, Frackowiak RSI, Grasby PM (1995): Dopaminergic modulation of impaired cognitive activation in the anterior cingulate cortex in schizophrenia. Nature 378:180-182

Endicott J, Spitzer RL (1978): A diagnostic interview: The schedule for affective disorders and schizophrenia. Arch Gen Psychiatry 35:837-844
Ernst M, Zametkin AJ, Matochik JA, Liebenauer LL, Fitzgerald GA, Cohen RM (1994): Effects of intravenous dextroamphetamine on brain metabolism in adults with attention-deficit hyperactivity disorder (ADHD). Preliminary findings. Psychopharmacol Bull 30:219-225

Foote SL, Freedman R, Oliver PA (1975): Effects of putative neurotransmitters on neuronal activity in monkey auditory cortex. Brain Res 86: 229-242

Friston KJ, Grasby PM, Bench CJ, Frith CD, Cowen PJ, Liddle PF, Frackowiak RSJ, Dolan RJ (1992): Measuring the neuromodulatory effects of drugs in man with positron emission tomography. Neurosci Lett 141:106-110

Gerfen CR (1992): The neostriatal mosaic: Multiple levels of compartmental organization in the basal ganglia. Annu Rev Neurosci 15:285-320

Glowinski J, Tassin JP, Theirry AM (1984): The mesocorticoprefrontal dopaminergic neurons. TINS 7:415-418

Grace AA (1995): The tonic/phasic model of dopamine system regulation: Its relevance for understanding how stimulant abuse can alter basal ganglia function. Drug Alcohol Depend 37:111-129

Huang SC, Phelps ME, Hoffman EJ, Sideris K, Selin CJ, Kuhl DE (1980): Noninvasive determination of local cerebral metabolic rate of glucose in man. Am J Physiol 238:E69E82

Johnson SW, Palmer MR, Freedman R (1983): Effects of dopamine on spontaneous and evoked activity of caudate neurons. Neuropharmacology 22:843-851

Kahn DA, Prohovnik I, Lucas LR, Sackeim HA (1989): Dissociated effects of amphetamine on arousal and cortical blood flow in humans. Biol Psychiatry 25:755-767

Kapur S, Meyer J, Wilson A, Houle S, Brown GM (1994): Modulation of cortical neuronal activity by a serotonergic agent: A PET study in humans. Brain Res 646:292294

Kim S-G, Ugurbil K, Strick PL (1994): Activation of a cerebellar output nucleus during cognitive processing. Science 265:949-951

Kuczenski R, Segal D (1989): Concomitant characterization of behavioral and striatal monoamine response to amphetamine using in vivo microdialysis. J Neurosci 9: 2051-2056

Leiner HC, Leiner AL, Dow RS (1993): Cognitive and language functions of the human cerebellum. TINS 16(11): $444-447$

Lou HC, Henriksen L, Bruhn P (1984): Focal cerebral hypoperfusion in children with dysphasia and/or attention deficit disorder. Arch Neurol 41:825-829

Lou HC, Henriksen L, Bruhn P, Borner H, Nielsen JB (1989): Striatal dysfunction in attention deficit and hyperkinetic disorder. Arch Neurol 46:48-52

Mantz J, Milla C, Glowinski J, Thierry AM (1988): Differential effects of ascending neurons containing dopamine and noradrenaline in the control of spontaneous activity and evoked responses in the rat prefrontal cortex. Neuroscience $27: 517-526$

Massion J, Rispal-Padel L (1986): The thalamus: Motor functions. Rev Neurol (Paris) 142:327-336

Mathew RJ, Wilson WH (1985): Dextroamphetamine- 
induced changes in regional cerebral blood flow. Psychopharmacology 87:298-302

Matochik JA, Nordahl TE, Gross M, Semple WE, King AC, Cohen RM, Zametkin AJ (1993): Effects of acute stimulant medication on cerebral metabolism in adults with hyperactivity. Neuropsychopharmacology 8:377-386

Matochik IA, Liebenauer LL, King AC, Szymanski HV, Cohen RM, Zametkin AJ (1994): Cerebral glucose metabolism in adults with attention deficit hyperactivity disorder after chronic stimulant treatment. Am J Psychiatry 151:658-664

Matsui T, Hirano A (1978): An Atlas of the Human Brain for Computerized Tomography. New York, Igaku-Shoin Medical

Mattay VS, Berman KF, Ostrem JL, Esposito G, Van Horn JD, Bigelo LB, Weinberger DR (1996): Dextroamphetamine enhances "neural network specific" physiological signals: A PET rCBF Study. J Neurosci 16:4816-4822

Metz JT, de Wit H, Cooper M. (1991): Amphetamine and regional cerebral metabolic rate: Effects of behavioral state and dose. J Cereb Blood Flow 11(suppl 2):S390

Mogenson GJ, Yim CC (1991): Neuromodulatory functions of the mesolimbic dopamine system: Electrophysiological and behavioral studies. In Willner P, Scheel-Kruger J (eds), The Mesolimbic Dopamine System. New York, Wiley, pp 105-113

Phelps ME, Huang SC, Hoffman EJ, Selin C, Sokoloff L, Kuhl DE (1979): Tomographic measurement of local cerebral glucose metabolic rate in humans with $\left[{ }^{18} \mathrm{~F}\right] 1$-fluoro-2deoxy-D-glucose: Validation of method. Ann Neurol 6:371-388

Porrino LJ, Lucignani G, Dow-Edwards D, Sokoloff L (1984): Correlation of dose-dependent effects of acute amphetamine administration on behavior and local metabolism in rats. Brain Res 307:311-320

Rapoport J, Buchsbaum MS, Weingartner H, Zahn TP, Ludlow C, Mikkelsen EJ (1980): Dextroamphetamine: Its cognitive and behavioral effects in normal and hyperactive boys and normal men. Arch Gen Psychiatry 37:933-943
Rumsey JM, Andreason P, Zametkin A, Aquino T, King AC, Hamburger SD, Pikus A, Rapoport JL, Cohen RM (1992): Failure to activate left temporoparietal cortex in dyslexia; ${ }^{15} \mathrm{O}$ PET study. Arch Neurol 49:545-552

Schmidt ME, Ernst M, Matochik JA, Maisog JM, Pan BS, Zametkin AJ, Potter WZ (1996): Cerebral glucose metabolism during pharmacologic studies. Test-Retest under placebo conditions. J Nucl Med 37:1142-1149

Sokoloff L (1984): Modeling metabolic process in the brain in vivo. Ann Neurol 15(suppl):S1-S11

Spielberger CD, Gorsuch RL, Lushene RE (1970): Manual for the State-Trait Anxiety Inventory. Palo Alto, CA, Consulting Psychologist Press

Sprouse JS, Aghajanian GK (1988): Responses of hippocampal pyramidal cells to putative serotonin 5-HT1A and 5-HT1B agonists: A comparative study with dorsal raphe neurons. Neuropharmacology 27:707-715

Tucker DM, Luu P, Pribram KH (1995): Social and emotional self-regulation. Ann NY Acad Sci 769:213-239

Wang GJ, Volkow ND, Fowler JS, Ferrieri R, Schlyer DJ, Alexoff D, Pappas N, Lieberman J, King P, Warner D, Wong C, Hitzemann RJ, Wolf AP (1994): Methylphenidate decreases regional cerebral blood flow in normal human subjects. Life Sci 54:PL 143-146

Wechsler LR, Savaki HE, Sokoloff L (1979): Effects of d- and l- amphetamine on local cerebral glucose utilization in the conscious rat. J Neurochem 32:15-22

Weiner N (1980): Norepinephrine, epinephrine, and the sympathomimetic amines. In Goodman AG, Gilman A (eds), The Pharmacological Basis of Therapeutics. New York, Macmillan, p 161

West WB, Van Groll BJ, Appel JB (1995): Stimulus effects of d-amphetamine II: DA, NE, and 5-HT mechanisms. Pharmacol Biochem Behav 51:69-76

Wolkin A, Angrist B, Wolf A, Brodie J, Wolkin B, Jaeger J, Cancro R, Rotrosen J (1987): Effects of amphetamine on local cerebral metabolism in normal and schizophrenic subjects as determined by positron emission tomography. Psychopharmacology 92:241-246 